\title{
TECNOLOGIAS VERDES DE APLICACIÓN EN ENVOLVENTES VERTICALES EN ZONAS ÁRIDAS. EVALUACIÓN ENERGÉTICA Y AMBIENTAL
}

\section{GREEN TECHNOLOGIES APPLIED IN VERTICAL ENVELOPES IN ARID CLIMATES. ENERGY AND ENVIRONMENTAL ASSESSMENT}

Presentación: 6 y 7 de octubre 2020.

\section{Doctorando:}

\author{
Pablo Suárez \\ Instituto de Ambiente, Hábitat y Energía (INAHE - CCT CONICET Mendoza), Consejo Nacional de Investigaciones Científicas y \\ Técnicas (CONICET) - Facultad Regional Mendoza, Universidad Tecnológica Nacional - Argentina. \\ suarezpablo91@gmail.com
}

Directora tesis doctoral:

Érica Correa

Dir. Beca Doctoral:

\section{Alicia Cantón}

\begin{abstract}
Resumen
La infraestructura verde constituye una estrategia de mitigación de las temperaturas urbanas y edilicias debido a la disminución de consumo energético en el ambiente construido, con su consecuente disminución de las emisiones de efecto invernadero. Este impacto positivo se ve acentuado bajo condiciones de climas áridos. En el presente informe se describen las etapas de la investigación referidas al estudio de los Sistemas de Enverdecimiento Vertical (SEV). Actualmente se han desarrollado dos etapas: una primera de análisis de antecedentes con el fin de conocer el estado del arte respecto al impacto del uso de la tecnología en la disminución de las temperaturas superficiales exteriores e interiores y el ahorro energético que conllevan en relación con el tipo de tecnología implementada y al clima; y una segunda etapa referida al desarrollo de experimentos a campo con el objeto de monitorear el comportamiento térmico de viviendas másicas con SEV respecto a una vivienda testigo de igual tipología y materialidad. A tal fin, se monitorearon, durante dos veranos consecutivos, dos casos de estudio: una vivienda con FVT en orientación este y una vivienda testigo. Se registraron datos de temperatura ambiente exterior e interior; superficial exterior e interior y radiación horizontal. Los resultados encontrados muestran disminuciones de hasta $3.1^{\circ} \mathrm{C}$ en la temperatura ambiente interior de las viviendas con FVT, de hasta $27.4{ }^{\circ} \mathrm{C}$ en muros exteriores y de $6.5^{\circ} \mathrm{C}$ en muros interiores. Estos resultados demuestran el potencial de la aplicación de esta estrategia en un clima árido.
\end{abstract}

Palabras clave: Zonas Áridas, Sistemas de Enverdecimiento Vertical, Arquitectura Bioclimática.

\begin{abstract}
Green infrastructure is a strategy to mitigate urban temperatures and buildings due to the decrease in energy consumption in the built environment, with its consequent decrease in greenhouse emissions. This positive impact is accentuated under arid climate conditions. This report describes the stages of research related to the study of Vertical Greenery Systems (VGS). Two stages have now been developed: a first background analysis in order to know the state of the art regarding the impact of the use of technology on the decrease of outdoor and indoor surface temperatures and the energy savings they entail in relation to the type of technology implemented and the climate; and a second stage related to the development of field experiments in order to monitor the thermal behavior of smaller homes with regard to a witness house of equal typology and materiality. To this end, two cases of study were monitored for two consecutive summers: a housing with FVT in east orientation and a witness dwelling. Outdoor and indoor ambient temperature data were recorded; surface exterior and interior and horizontal radiation. The
\end{abstract}


results found show decreases of up to $3.1{ }^{\circ} \mathrm{C}$ in the indoor ambient temperature of FVT dwellings, up to $27.4{ }^{\circ} \mathrm{C}$ in exterior walls and $6.5^{\circ} \mathrm{C}$ in interior walls. These results demonstrate the potential of the implementation of this strategy in an arid climate.

Keywords: Arid Zones, Vertical Greening Systems, Bioclimatic Architecture.

\section{Introducción}

En áreas densamente pobladas existen impactos negativos propios del avance de la urbanidad sobre el medio ambiente; desde emisiones de carbono y el incremento de las temperaturas medias del aire, hasta la depredación de zonas productivas periféricas y la destrucción de los ecosistemas. De acuerdo a datos de la Agencia Internacional de Energía (OCED / IEA, 2017), las ciudades ocupan el $3 \%$ de la superficie del planeta y, además de provocar el aumento de las temperaturas medias del aire, son responsables del $67 \%$ del consumo energético global. A su vez, el Programa de Naciones Unidas para el Medio Ambiente (PNUMA) indica que el 75\% de la infraestructura que existirá en el 2030, aún no fue construida. Esto representa una oportunidad para crear "ciudades limpias y verdes", eficientes y resilientes. Una estrategia para alcanzar este objetivo es el enverdecimiento urbano.

La infraestructura verde genera beneficios energético-ambientales, a escala urbana, disminuye la isla de calor y aumenta el confort de los espacios públicos $\mathrm{y}$, a escala edilicia, reduce el consumo de energía para acondicionamiento de los espacios interiores. Investigaciones recientes han determinado que en un clima templado el aumento de la infraestructura verde en un $10 \%$ podría reducir las temperaturas medias del aire urbano $2.5{ }^{\circ} \mathrm{C}$ (Gill et al., 2007) y que en un clima seco árido se puede disminuir $3.8^{\circ} \mathrm{C}$ la temperatura ambiente en un lugar arbolado (Goretti Salas Esparza, 2017). Además, los espacios verdes generan beneficios para la salud y bienestar de los ciudadanos (Contesse et al., 2018). Dado que el fenómeno de consolidación de las ciudades ha limitado el potencial de incorporación de estructuras verdes tradicionales - parques, plazas, arbolado de alineación-, han surgido nuevas tipologías de vegetación asociadas a cubiertas y muros verdes.

El desarrollo de conocimiento vinculado a los Sistemas de Enverdecimiento Vertical (SEV) ha crecido en función de su relevancia a nivel mundial en la última década (Bustami et al., 2018). Muestran probada eficiencia en la disminución de las temperaturas de los espacios habitables y sus consecuentes impactos en los consumos de energía. Los resultados varían en su magnitud según el tipo de clima donde se aplican los SEV, registrando máximas disminuciones de temperatura superficial exterior del orden de los $34{ }^{\circ} \mathrm{C}$ (Suklje et al., 2016) en climas del tipo $\mathrm{Cfa/Cfb}$ (templado cálidos húmedos, verano caliente), de temperatura ambiente interior del orden de los 5 - C (Haggag et al., 2014) en climas del tipo BWh (árido desértico, verano caliente) y de temperatura ambiente exterior del orden de los $3.3{ }^{\circ} \mathrm{C}(\mathrm{N}$. H. Wong et al., 2010) en climas del tipo Af (Ecuatorial húmedo). Mayoritariamente los estudios se han realizado en países europeos, asiáticos y norteamericanos; en climas del tipo templado cálido, tanto húmedo como seco -Csa, $\mathrm{Cfa} / \mathrm{Cfb}$-. Los resultados han mostrado similares o mejores rendimientos para los $\mathrm{SEV}$, en cuanto a las reducciones de temperatura, en climas áridos respecto a climas húmedos.

El Área Metropolitana de Mendoza, Argentina (AMM), presenta un clima árido desértico (BWk - Köppen-Geiger). Desde el punto de vista de la presencia de espacios vegetados, cuenta en su estructura con una importante cantidad de espacios abiertos forestados. Sin embargo, el proceso de crecimiento y densificación urbana no ha sido acompañado por un proceso de densificación del verde urbano y, además, ha limitado la disponibilidad de vacíos urbanos que permiten la incorporación de espacios verdes tradicionales. En consecuencia, incrementar las áreas verdes implica implementar nuevas tecnologías de vegetación como lo son, entre otras, los SEV.

\section{Desarrollo}

\section{Revisión bibliográfica}

La búsqueda bibliográfica se orientó a identificar documentos actualizados que abordan investigaciones de carácter científico referido al impacto de los SEV desde el punto de vista de su influencia en las condiciones termo-energéticas de los espacios interiores conexos y su impacto sobre el microclima del entorno. En relación a ello, se identificaron artículos científicos que abordan el estudio de la temática en diferentes localizaciones geográficas y climáticas, en el periodo 2005-2020. Los artículos recopilados fueron seleccionados en función de la calidad de estos, determinada a partir del análisis sistémico de indicadores (Saracevik, 1999). De esta forma, quedó definido un conjunto de 65 trabajos que abordan el comportamiento de SEV. Los contenidos fueron agrupados de acuerdo con los siguientes 
indicadores: Distribución geográfica y climática, distribución temporal de la producción, tipologías de SEV analizadas y resultados obtenidos.

Los resultados obtenidos fueron analizados en función de cuatro variables: diferencias de temperatura superficial entre el caso testigo y caso con SEV, diferencias de temperatura interior, diferencias de temperatura exterior y reducción del consumo energético para acondicionamiento de espacios interiores.

\section{Evaluación térmica: casos de estudio}

Esta segunda etapa consiste en evaluar el impacto de los SEV con mayor grado de utilización en la Ciudad de Mendoza: Las Fachadas Verdes Tradicionales (FVT). Además, es la estrategia de menor costo y dificultad de implementación. Respecto a la orientación, se buscaron casos con orientación este y oeste, dado que son los planos verticales que mayor radiación reciben en esta localización geográfica. Se seleccionan dos viviendas unifamiliares de tipología seriada en Guaymallén, Mendoza. Son del tipo morfológico de casa compacta, ampliada, estructurada en dos niveles. Tecnológicamente muestran el tipo constructivo característico de áreas sísmicas: estructura de hormigón armado y mampostería de ladrillo visto. Las cubiertas son planas, conformadas por losas de hormigón alivianadas, aisladas térmica e hidráulicamente. Una de ellas presenta el sistema de FVT en la fachada interna de la vivienda orientada al Este y en la fachada lateral orientada al norte. La FVT está conformada por una enredadera Parthenocisus Quinquefolia, vulgarmente denominada Parra Virgen- de un espesor promedio de $40 \mathrm{~cm}$ que cubre el $100 \%$ de la fachada este (Figura 1).

$$
\mid
$$

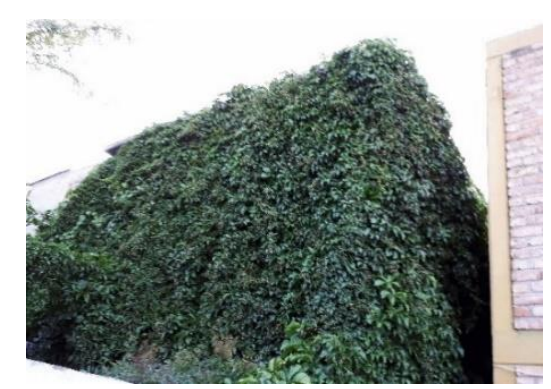

Vivienda con FVT

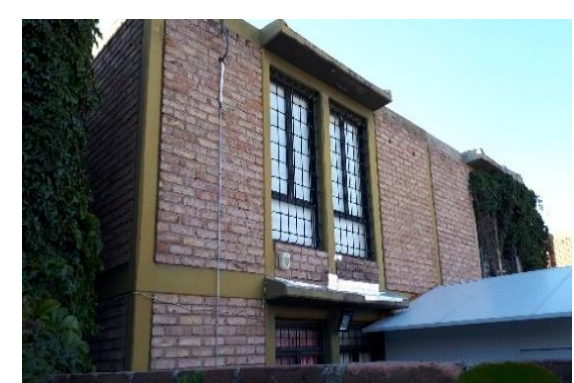

Vivienda testigo

Figura 1: Casos de estudio.

Para la evaluación de la incidencia de la FVT en el comportamiento térmico de las fachadas de orientación este de las viviendas, las variables medidas fueron: temperatura y humedad del aire exterior en los espacios abiertos públicos y privados colindantes con la vivienda, temperatura y humedad del aire interior y temperatura superficial interior y exterior en muros. Los sensores utilizados para medir la temperatura y humedad relativa ambiente fueron del tipo termistor y termocupla. La toma de datos se registró en dos períodos: verano 2019 y 2020 durante 15 días en cada uno de ellos.

\section{Resultados}

\section{Revisión bibliográfica}

La bibliografía recopilada muestra que el origen geográfico de los documentos analizados mayoritariamente proviene del continente europeo -64\%- y, en menor proporción, de países asiáticos -21\%-y sudamericanos -14\%-. (Figura 2)

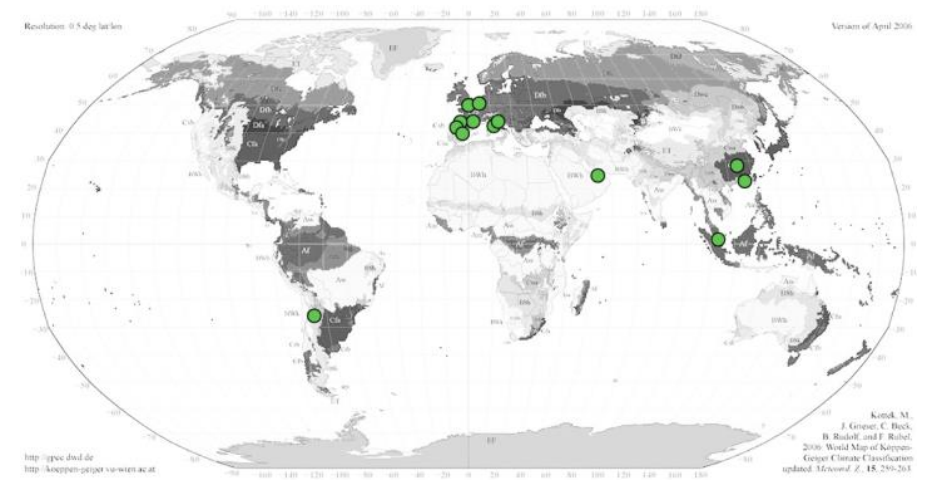

Figura 2: Distribución geográfica de documentos analizados 
El desarrollo de conocimiento se registra mayoritariamente en climas del tipo Cfa/Cfb (templado cálido húmedo, verano caliente) y del tipo Csa (templado cálido, verano seco y caliente); y en menor medida para climas del tipo CWb (templado cálido desértico, verano caliente), Af (Ecuatorial húmedo) y BWh (árido desértico).

En el período 2005 - 2020 se observa una tendencia de crecimiento positiva. Durante la última década, la producción se ha incrementado 2.5 veces, es decir, creció del 10\% en el año 2010 al 25\% en el año 2017 (Figura 3). Sin embargo, se observa una leve desaceleración de la temática a partir del año 2019.

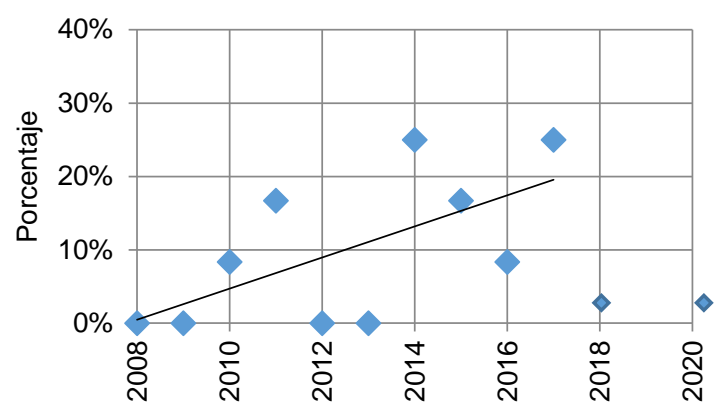

Período 2008-2020

Figura 3: Distribución geográfica de documentos analizados

Los SEV se agrupan en dos categorías: Pared Viva (PV) y Fachadas Verdes (FV) (Figura 1). Las FV son aquellos sistemas en los que hay plantas trepadoras y/o arbustos colgantes cubriendo un área determinada. Pueden dividirse en tres tipologías: Fachadas Verdes Tradicionales (FVT), donde la planta se adosa al muro del edificio; Fachadas Verdes tipo Doble Piel (FVDP) o cortina verde, donde existe una estructura suplementaria que se separa una distancia variable de la pared del edificio; y Fachadas Verdes con Macetas Perimetrales (FVMP), donde pueden alojarse macetas con plantas trepadoras y/o arbustos colgantes para generar una cortina verde. Dentro del conjunto de estudios analizados, las Paredes Vivas son el SEV en el cual se han centrado las investigaciones durante la última década. Del universo de documentos revisados, el 50\% corresponde a Paredes Vivas, el $41 \%$ a Fachadas Verdes y el 9\% a Techos Verdes. Dentro del sistema denominado Paredes Vivas, las tipologías de paneles modulares presentan la mayor cantidad de casos estudiados. Y en el sistema denominado Fachadas Verdes, la más estudiada corresponde a la tipología de doble piel.

Los beneficios termo-energéticos han sido analizados ampliamente en la literatura internacional. Sin embargo, es acotado el desarrollo de conocimiento en relación con la incidencia de las FVT en el comportamiento térmico de los espacios interiores en climas del tipo desértico y al análisis del impacto diferencial del uso de la estrategia de acuerdo con la orientación de la fachada.

\section{Evaluación térmica: casos de estudio}

A fin de reconocer potenciales impactos del uso de FVT en muros exteriores de mampostería con exposición este, se compararon las temperaturas superficiales exteriores, superficiales interiores y ambiente interior de las dos viviendas monitoreadas. La máxima reducción de temperatura superficial exterior fue de $27.4{ }^{\circ} \mathrm{C}$ (Figura 4). Los picos de diferencia de temperatura se observan en ambos períodos a las 10:45 am, en días de cielo claro. Esto pone de manifiesto que la temperatura superficial exterior es fundamentalmente comandada por el fenómeno radiativo, teniendo en cuenta la orientación del muro -este-. Los resultados son superiores a los encontrados por Vox et al. (2018) y Hoelscher et al. (2016), las cuales oscilan entre $9.0^{\circ} \mathrm{C}$ y $15.5^{\circ} \mathrm{C}$ (FVT en orientación Norte y en clima del tipo Csa, mediterráneo, FVT en orientación nor-oeste y clima del tipo Dfb, templado cálido, respectivamente).

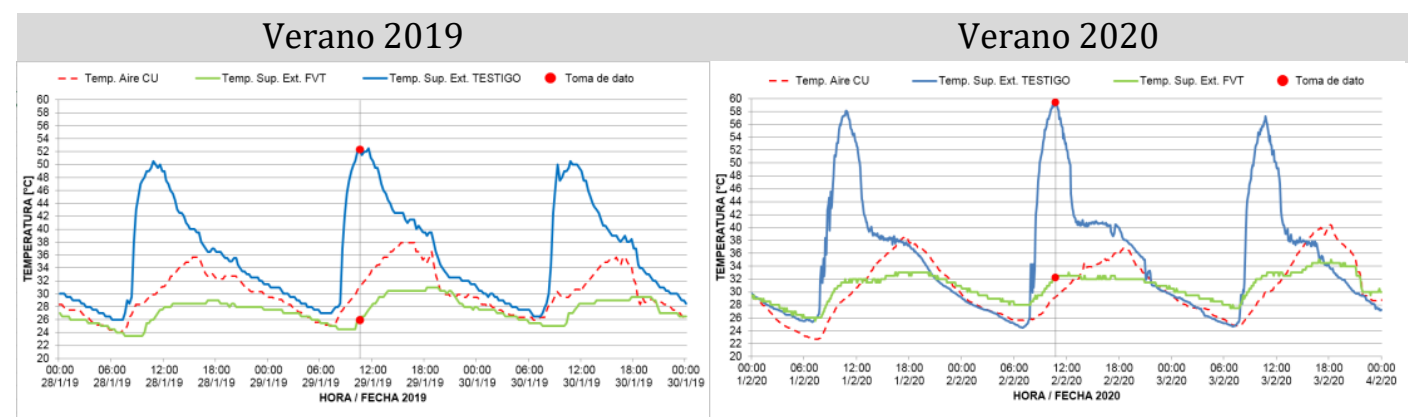

Figura 4: Temperatura superficial exterior: caso testigo y caso con FVT, orientación este. 
El caso con FVT muestra temperaturas superficiales interiores menores, respecto del caso testigo con una reducción máxima de $6.5^{\circ} \mathrm{C}$. Estas diferencias de temperatura se observan en ambos períodos entre las 14:45 y las 15:45, una y dos horas después del mediodía solar (Figura 5). Los resultados son superiores a las magnitudes encontradas por Hoelscher et al. (2016) y Susorova et al. (2013), los cuales oscilan entre $1.7^{\circ} \mathrm{C}$ y $2.0^{\circ} \mathrm{C}(\mathrm{FVT}$ en orientación Oeste y en clima del tipo Dfb templado cálido, FVT en orientación Norte y en clima del tipo Dfa, continental verano caliente, respectivamente).
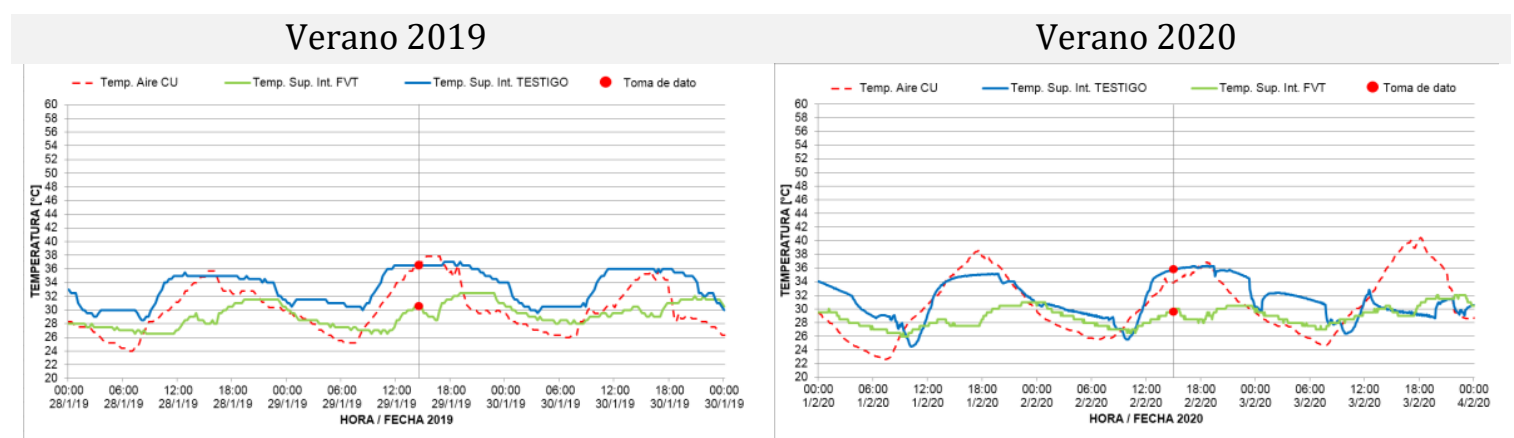

Figura 5: Temperatura superficial interior: caso testigo y caso con SEV, en orientación este.

El análisis de la temperatura ambiente interior identifica una máxima diferencia de $3.1^{\circ} \mathrm{C}$ (Figura 6). Al igual que en lo observado en el análisis de las temperaturas superficiales, las diferencias son superiores a las encontradas por Kontoleon \& Eumorfopoulou (2010) que fueron del orden de $0.5^{\circ} \mathrm{C}$ (FVT en orientación este y en clima del tipo tipo $\mathrm{Cfb}$, oceánico templado verano suave). Mientras que Hoelscher et al., (2016) y Perini et al., (2011) no encontraron efectos del FVT sobre la temperatura del aire en espacios interiores. Estos resultados pueden explicarse por la utilización de FVT menores a $20 \mathrm{~cm}$ de espesor, en orientación Nor-Oeste.
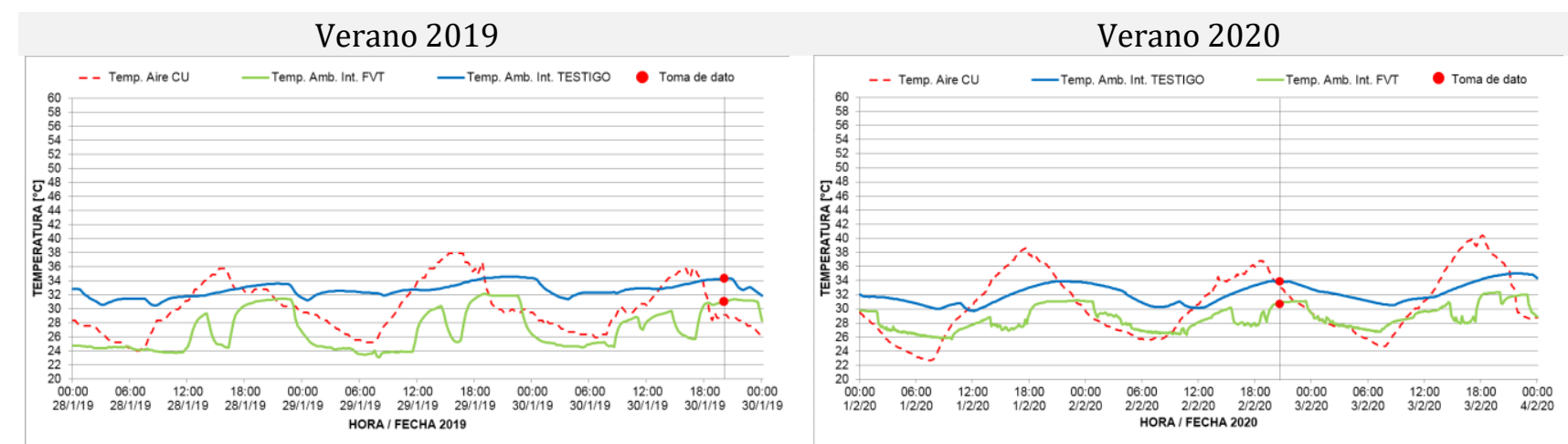

Figura 6: Temperatura ambiente interior: caso testigo y caso con SEV, en orientación este

\section{Conclusiones}

Los resultados encontrados son alentadores; en primera instancia por haber hallado, en la literatura internacional, que la magnitud del beneficio térmico del uso de los SEV es mayor en climas áridos; y en segunda instancia porque se ha verificado en la evaluación de las viviendas que las magnitudes son contrastables con los resultados encontradas por otros autores. A su vez, estos resultados son relevantes en cuanto al valor del uso de la estrategia para disminuir las temperaturas interiores y aproximar a las condiciones de confort a los espacios habitables dentro de los edificios en climas de condición árida.

Los resultados pormenorizados del análisis crítico del estado del arte se desarrollan en Suárez et. al. (2018). Los resultados de la evaluación térmica de casos reales se encuentran publicados por Suárez et. al. (2019 a, b; 2020).

\section{Referencias}

Alexandri, E., \& Jones, P. (2008). Temperature decreases in an urban canyon due to green walls and green roofs in diverse climates. Building and Environment, 43(4), 480-493. Recuperado de

https://doi.org/10.1016/j.buildenv.2006.10.055 
Bustami, R. A., Belusko, M., Ward, J., \& Beecham, S. (2018). Vertical greenery systems : A systematic review of research trends. Building and Environment, 146(August), 226-237. Recuperado de https://doi.org/10.1016/j.buildenv.2018.09.045

Contesse, M., van Vliet, B. J. M., \& Lenhart, J. (2018). Is urban agriculture urban green space? A comparison of policy arrangements for urban green space and urban agriculture in Santiago de Chile. Land Use Policy, 71(October), 566577. Recuperado de https://doi.org/10.1016/j.landusepol.2017.11.006

Gill, S. E., Handley, J. F., Ennos, A. R., \& Pauleit, S. (2007). Adapting cities for climate change: The role of the green infrastructure. Built Environment, 33(1), 115-133. Recuperado de https://doi.org/10.2148/benv.33.1.115

Goretti Salas Esparza, M. (2017). La vegetación como sistema de control para las islas de calor urbano en ciudad Juárez, Chihuahua. Revista Hábitat Sustentable, 7, Págs. 14-23. Recuperado de https://doi.org/10.22320/07190700.2017.07.01.02

HAGGAG, M., HASSAN, A., \& ELMASRY, S. (2014). Experimental study on reduced heat gain through green façades in a high heat load climate. Energy and Buildings, 82, 668-674. Recuperado de https://doi.org/10.1016/j.enbuild.2014.07.087

HOELSCHER, M. T., NEHLS, T., JÄNICKE, B., \& WESSOLEK, G. (2016). Quantifying cooling effects of facade greening: Shading, transpiration and insulation. Energy and Buildings, 114, 283-290. Recuperado de https://doi.org/10.1016/j.enbuild.2015.06.047

KONTOLEON, K. J., \& EUMORFOPOULOU, E. A. (2010). The effect of the orientation and proportion of a plant-covered wall layer on the thermal performance of a building zone. Building and Environment, 45(5), 1287-1303. Recuperado de https://doi.org/10.1016/j.buildenv.2009.11.013

OCED / IEA. (2017). Energy Technology Perspectives 2017. In International Energy Agency (IEA) Publications. Recuperado de https://webstore.iea.org/download/summary/237?fileName=English-ETP-2017-ES.pdf

PERINI, K., OTTELÉ, M., FRAAIJ, A. L. A., HAAS, E. M., \& RAITERI, R. (2011). Vertical greening systems and the effect on air flow and temperature on the building envelope. Building and Environment, 46(11), 2287-2294. Recuperado de https://doi.org/10.1016/j.buildenv.2011.05.009

SUKLJE, T., SASO, M., \& ARKAR, C. (2016). On detailed thermal response modeling of vertical greenery systems as cooling measure for buildings and cities in summer conditions. 115, 1055-1068. Recuperado de https://doi.org/10.1016/j.energy.2016.08.095

SUSOROVA, I., ANGULO, M., BAHRAMI, P., \& BRENT STEPHENS. (2013). A model of vegetated exterior facades for evaluation of wall thermal performance. Building and Environment, 67, 1-13. Recuperado de https://doi.org/10.1016/j.buildenv.2013.04.027

VOX, G., BLANCO, I., \& SCHETTINI, E. (2018). Green façades to control wall surface temperature in buildings. Building and Environment, 129(September 2017), 154-166. Recuperado de https://doi.org/10.1016/j.buildenv.2017.12.002

WONG, I., \& BALDWIN, A. N. (2016). Investigating the potential of applying vertical green walls to high-rise residential buildings for energy-saving in sub-tropical region. Building and Environment, 97, 34-39. Recuperado de https://doi.org/10.1016/j.buildenv.2015.11.028

WONG, N. H., KWANG TAN, A. Y., TAN, P. Y., CHIANG, K., \& WONG, N. C. (2010). Acoustics evaluation of vertical greenery systems for building walls. Building and Environment, 45(2), 411-420. Recuperado de https://doi.org/10.1016/j.buildenv.2009.06.017. 